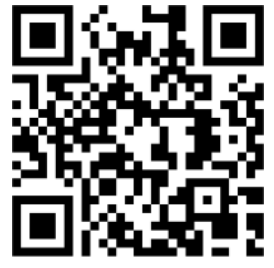

http://www.seer.ufm s.br/index.php/pecib es/index

*Autor

correspondente:

Angélica Pereira de

Arruda, Universidade

Federal de Mato

Grosso do Sul

- UFMS.

E-mail do autor: angelicafisio13@gmai 1.com

Palavras-chave:

Extubação. Lesões encefálicas. Desmame do Respirador. Unidades de Terapia Intensiva.

Key-words: Extubation. Brain Injuries. Ventilator Weaning, Intensive Care Units.

\section{Avaliação do escore visage como preditor de sucesso de extubação em pacientes com lesão cerebral aguda}

The evaluation of the visage score as a predictor of extubation success in patients with acute brain injury

Angélica Pereira de Arruda ${ }^{1}$, Flávia Manhani Muzette ${ }^{2}$, Karla Luciana Magnani Seki ${ }^{3}$

${ }^{1}$ Fisioterapeuta, pós graduanda pelo programa de residência multiprofissional em atenção ao paciente crítico pela Universidade Federal de Mato Grosso do Sul - UFMS

${ }^{2}$ Fisioterapeuta, mestre pela Universidade Federal de Mato Grosso do Sul - UFMS

${ }^{3}$ Fisioterapeuta, coordenadora do programa de residência multiprofissional- COREMU, docente na Universidade Federal de Mato Grosso do Sul - UFMS

\section{Resumo}

Objetivo: Avaliar a associação do Escore VISAGE como fator preditivo de sucesso na extubação dos pacientes com lesão cerebral aguda grave, correlacionando os resultados obtidos do Escore com o desfecho e comparação de algumas variáveis clínicas dos grupos estudados. Metodologia: Trata-se de um estudo transversal e observacional, desenvolvido em uma Unidade de Terapia Intensiva do Hospital Santa Casa de Campo Grande. Foram avaliados 64 pacientes com traumatismo cranioencefálico e acidente vascular cerebral, diagnosticados e confirmados pela história clínica e da tomografia computadorizada de crânio, de ambos os sexos, intubados por 48h e elegíveis para o teste de respiração espontânea. Resultados: Dos 64 pacientes selecionados (47 homens, média de idade 50,5 anos), a extubação foi bem- sucedida em 48 casos. O grupo sucesso na extubação apresentou escore total do VISAGE maior que o grupo falha $(\mathrm{p}=0,031)$. Na comparação dos domínios do escore VISAGE, entre os dois grupos estudados, houve diferença significativa no domínio deglutição $(\mathrm{p}=0,007)$. O grupo falha na extubação apresentou maior tempo de internação na Unidade de Terapia Intensiva $(p=0,002)$. Conclusões: Na amostra de pacientes neurocríticos estudados, valores superiores da pontuação total do escore de VISAGE estiveram associados com o sucesso na extubação, sendo a deglutição um importante fator a ser considerado.

\section{Abstract}

Objective: To evaluate the association of the VISAGE score as a predictor of success in the extubation of patients with severe acute brain injury, correlating the results obtained from the score with the outcome and comparison of some clinical variables in the studied groups. Methodology: It is a cross-sectional observational study, developed in an Intensive Care Unit of Hospital Santa Casa de Campo Grande. Sixty-four patients with traumatic brain injury and stroke were avaluated, diagnosed and confirmed by clinical history and cranial computed tomography, of both genders, intubated for 48 hours and eligible for the spontaneous breathing test. Results: From the 64 selected patients (47 men, mean age 50.5 years), the extubation procedure was successful in 48 cases. The extubation success group had a higher total VISAGE score than the failure group $(p=0.031)$. When comparing the domains of the VISAGE score between the two groups studied, there was a significant difference in the domain of swallowing $(\mathrm{p}=0.007)$. The extubation failure group had a longer stay in the Intensive Care Unit $(\mathrm{p}=0.002)$. Conclusions: In the sample of neurocritical patients evaluated, the higher values of the total score of the VISAGE score were associated with successful extubation, with swallowing being an important factor to be considered. 


\section{Introdução}

A falha na extubação de pacientes submetidos a ventilação mecânica $(\mathrm{VM})$ em uma unidade de terapia intensiva (UTI) varia em torno de $2-25 \%$, sendo necessário realizar a reintubação, porém, essas taxas podem mudar de acordo com o tipo de paciente e protocolo de desmame usado $^{8}$. No que se refere a extubação no paciente neurocrítico, de acordo com a literatura, a taxa de falha de extubação nesta população é de 20 a $40 \%$ nas UTI's neurológicas. ${ }^{6,11}$

Apesar das altas taxas de falha, ainda são insuficientes as informações e estudos disponíveis sobre o desmame da VM e extubação de pacientes neurocríticos. Os protocolos disponíveis na literatura são referentes a população sem patologia neurológica grave, que não são comparáveis com os indivíduos com traumatismo craniano grave (TCE) ou com acidente vascular cerebral (AVC), por diversas razões. ${ }^{9}$

Uma das razões é que o paciente neurocrítico em ventilação mecânica, em geral, não é intubado por insuficiência respiratória primária, mas sim devido ao seu estado de consciência diminuído e incapacidade de proteção das vias aéreas, de modo que o objetivo principal da via aérea artificial é prevenir danos secundários como a hipoxemia, hipercapnia e hipocapnia. ${ }^{5}$

Outra razão, é que normalmente estes indivíduos são submetidos a um tempo maior de VM e de uso de vias aéreas artificiais comparados a indivíduos sem lesão neurológica grave, e com isso o paciente neurocrítico pode apresentar vários graus de comprometimento do nível de consciência ao ser retirado do suporte ventilatório, seguindo muitas vezes com incapacidade de seguir comandos e de realizar manobras para obter parâmetros clássicos de desmame da VM e/ou extubação. ${ }^{14}$

A extubação de pacientes neurocríticos continua sendo um desafio, pois estudos que investigam fatores preditivos claros e específicos para essa população ainda são poucos. Como consequência, nenhuma recomendação, protocolo ou diretriz existe para esta população específica e as variações de prática são frequentes nos hospitais. ${ }^{11,17}$

Contudo, em 2017 foi realizado um estudo multicêntrico prospectivo que determinou, por meio de análises multivariadas, os preditores de extubações bemsucedidas em indivíduos com danos cerebrais graves e a partir deste estudo foi criado o escore VISAGE ${ }^{3}$. O escore combina sinais clínicos de fácil avaliação e aplicação, que pode ser facilmente realizado a beira leito, tais como a capacidade do individuo de realizar busca visual, a capacidade de deglutição, o nível de consciência, conforme a escala de coma de glasgow e a idade ${ }^{3,6,11,18}$. De acordo com os autores, a avaliação do escore pode ajudar a predizer o sucesso de extubação em individuos neurocriticos que obtivessem pontuação total maior ou igual a tres pontos no escore VISAGE.

Assim, o presente estudo teve como objetivo avaliar a a associação do escore VISAGE no processo de desmame ventilatório e relacioná-lo com o desfecho da extubação em uma unidade de terapia intensiva neurológica.

\section{Material e Métodos}

Trata-se de um estudo transversal e observacional. A pesquisa foi realizada em uma Unidade de Terapia Intensiva (UTI) Adulto do Hospital Santa Casa de Campo Grande, Mato Grosso do Sul, no período de janeiro a agosto de 2020. O presente projeto de pesquisa foi aprovado pelo comitê de ética e pesquisa da Universidade Federal do Mato Grosso do Sul, sob o parecer de número 3.621.431

\subsection{Participantes}

A amostra de estudo foi composta por 64 indivíduos adultos (com idade superior a 18 anos), de ambos os sexos, com lesão cerebral aguda, diagnosticados e confirmados pela história clínica e da tomografia computadorizada de crânio (TCC), sendo 35 pacientes diagnosticados com TCE e 29 com AVC. Todos os indivíduos incluídos no estudo respeitaram os critérios de inclusão/exclusão estabelecidos na pesquisa e os responsáveis dos mesmos estavam de acordo com o termo de consentimento livre e esclarecido (TCLE).

Foram incluídos indivíduos com lesão cerebral aguda, intubados; em ventilação mecânica invasiva no mínimo por $48 \mathrm{~h}$, estáveis hemodinamicamente e candidatos ao desmame ventilatório. Não participaram da pesquisa pacientes com evidência de lesão cerebral crônica preexistente; presença de neoplasia cerebral; sequelas motoras prévias; indivíduos em investigação ou confirmação de morte encefálica; alta ou óbito com menos de 48 h na UTI; indivíduos com lesão medular; estado epiléptico; traqueostomizados; aqueles que se extubaram acidentalmente, gestantes, indígenas e quilombolas.

\subsubsection{Parâmetros avaliados}

Os pacientes foram triados nas primeiras 24 horas de admissão na UTI. Foram selecionados pela pesquisadora os indivíduos que se enquadraram nos critérios de inclusão do estudo. Durante a visita dos familiares à UTI foi esclarecido aos responsáveis do paciente sobre os objetivos da pesquisa, bem como os aspectos éticos da Resolução 466/12 do Ministério da Saúde.

Àqueles que aceitaram participar do estudo e que estavam de acordo com os critérios de inclusão propostos na pesquisa foi solicitada a assinatura do termo de consentimento livre e esclarecido. Logo após foram coletados os dados gerais dos participantes por uma ficha previamente elaborada, contendo informações pessoais, informações pertinentes ao quadro clínico, presença de morbidade e/ou comorbidade prévia, diagnóstico, pontuação do índice de gravidade do paciente (APACHE II), escala de coma de Glasgow (ECG), tempo de sedação, data da extubação, data de alta na unidade de terapia intensiva (UTI), entre outros. Todas essas informações eram colhidas no sistema de prontuários do hospital e preenchidas na admissão e na alta do participante da UTI.

Após a resolução das disfunções orgânicas agudas, quando realizado o despertar, foi realizada a elegibilidade para o teste de respiração espontânea (TRE) pelos profissionais de saúde do setor, sem nenhuma interferência dos pesquisadores. Somente durante o período de desmame 
da ventilação mecânica foi então aplicado o Escore VISAGE. A avaliação do escore foi realizada à beira leito, antes da extubação e quando o paciente era considerado elegível para o teste do TRE, porém, antes de ser realizado o mesmo. O escore VISAGE, desenvolvido por Asehnoune et al.9, inclui a pontuação da ECG, a idade do participante, a capacidade de resposta ocular ao perseguir uma caneta ou o próprio dedo do pesquisador e a avaliação da capacidade de deglutição (era apenas observado se o paciente era capaz de iniciar o movimento de deglutir ao comando do pesquisador) e para cada domínio avaliado era considerada uma pontuação de 0 ou 1 pontos, sendo a pontuação máxima de quatro (4) pontos.

As avaliações realizadas não interferiram na decisão da equipe que realizaria a extubação. A variável estudada, após a aplicação do escore foi o desfecho de sucesso ou insucesso da extubação, após a primeira tentativa. A definição de falha na extubação foi considerada quando houve necessidade de reintubação nas primeiras $48 \mathrm{~h}$ em respiração espontânea. ${ }^{4}$

\subsubsection{Análise de dados}

As variáveis contínuas e categóricas foram expressas em média \pm desvio padrão da média, frequência relativa ou mediana (intervalos interquartis). No primeiro momento foi aplicado o teste de ShapiroWilk para identificação dos dados paramétricos ou nãoparamétricos.

A análise inferencial foi realizada pela comparação dos desfechos do processo do desmame do suporte ventilatório e da extubação entre os grupos sucesso versus falha da extubação. As variáveis relativas às informações gerais e sóciodemográficas, nível de consciência e o escore total do VISAGE foram analisadas por meio do teste $\mathrm{t}$ Student não pareado ou teste $\mathrm{U}$ de Mann Whitney.

O nível de consciência também foi comparado intra-grupo, a fim da identificação do ganho ou não da pontuação da ECG inicial da admissão hospitalar versus a ECG no momento da realização dos testes avaliativos em ambos os grupos, por meio do teste t Student pareado ou teste de Wilcoxon.

Além dos dados categóricos gerais, como sexo, comorbidade, tipo de tratamento e causa da lesão, também, foram comparadas as pontuações de cada um dos domínios do escore VISAGE dos dois grupos estudados, por meio do teste qui-quadrado. A análise estatística foi realizada através do programa estatístico Graphpad Prism, versão 6.0, adotando nível de significância de 5\%.

\section{Resultados.}

Dos 644 pacientes admitidos na UTI em que a pesquisa foi realizada, 457 eram pacientes neurológicos. Destes 457 pacientes, 393 não foram incluídos no estudo por não respeitarem os critérios de inclusão e no total 64 pacientes foram incluídos no estudo.

A média de idade total dos participantes foi de 50,5 anos. Houve predomínio do sexo masculino, $\mathrm{n}=47$ $(73,44 \%)$. O diagnóstico clínico mais comum foi o de traumatismo cranioencefálico, em 35 pacientes $(54,69 \%)$, seguido de acidente vascular encefálico, $n=29(45,32 \%)$. Dos indivíduos que foram diagnosticados com TCE, a principal causa do trauma foi devido a queda, $\mathrm{n}=15(42,85 \%)$, seguido de acidente automobilístico, $\mathrm{n}=11(31,42 \%)$. A média de idade nessa mesma população foi de 41 anos, com predominância expressiva do sexo masculino, $n=31(89 \%)$. Entre os pacientes avaliados que receberam o diagnóstico de AVC, a média de idade foi maior (60 anos), mostrando uma diferença estatística de p <0,0001. Também houve predominância do sexo masculino, $\mathrm{n}=16(55 \%)$, apresentando diferença estatística de p 0,0026, e a principal causa do AVC foi a hemorrágica, $\mathrm{n}=22(75,86 \%)$. Os resultados da idade e sexo segundo o diagnóstico, são encontrados na Tabela 1.

Tabela 1 - Idade e sexo segundo o diagnóstico de Traumatismo Cranioencefálico e Acidente Vascular Cerebral.

\begin{tabular}{llll}
\hline Variável & TCE & AVC & P \\
\hline Idade & $41[28-58]$ & $60[56-69]$ & $<0,0001^{*}$ \\
Sexo & & & \\
Masculino & $31(89 \%)$ & $16(55 \%)$ & $0,0026^{*}$ \\
Feminino & $04(11 \%)$ & $13(45 \%)$ & \\
\hline
\end{tabular}

TCE: Traumatismo Cranioencefálico, AVC: Acidente Vascular Cerebral. Os resultados estão expressos em média \pm desvio padrão da média ou mediana (intervalos interquartis). Teste Mann- Whitney e teste qui-quadrado.

Os participantes do estudo foram divididos em grupos 2 grupos: grupo sucesso e grupo falha. Quarenta e oito pacientes $(75,0 \%)$ apresentaram extubação bem-sucedida e 16 pacientes $(25,0 \%)$ apresentaram falha de extubação. A principal causa de falha na extubação foi a inabilidade de manter a proteção da via aérea, com $n=9(56,25 \%)$. Os dados gerais dos participantes são mostrados na tabela 2. Pôde-se observar que houve diferença significativa apenas para a variável tempo de internação na Unidade de Terapia Intensiva (UTI), sendo observado tempo superior no grupo de falha da extubação $(\mathrm{p}=0,002)$. 
Tabela 2 - Dados gerais dos grupos dos dois grupos estudados.

APACHE II: Acute Physiology and Chronic Health Evaluation II;

\begin{tabular}{lrrr}
\hline \multicolumn{1}{c}{ Variáveis } & \multicolumn{2}{c}{ Grupos } & P \\
\cline { 2 - 3 } & Sucesso (n=48) & Falha (n=16) & \\
\hline Idade (anos) & $49,0 \pm 19,0$ & $58,0 \pm 13,5$ & 0,105 \\
APACHE II (pontos) & $21,5 \pm 5,0$ & $21,5 \pm 6,0$ & 0,893 \\
Tempo de sedação (dias) & $3,0 \pm 2,0$ & $3,0 \pm 1,5$ & 0,713 \\
Tempo de TOT (dias) & $7,0 \pm 2,5$ & $6,0 \pm 2,5$ & 0,283 \\
Tempo de internação (dias) & $9,0 \pm 3,0$ & $12,0 \pm 4,5$ & $0,002^{*}$ \\
UTI & & & \\
\hline
\end{tabular}

TOT: Tubo Orotraqueal; UTI: Unidade de Terapia Intensiva. Osresultados estão apresentados em média \pm desvio padrão da média. Teste T Student não pareado.

A Tabela 3 apresenta os resultados encontrados na comparação intragrupo das pontuações da ECG inicial (no momento da admissão hospitalar) versus no momento da avaliação do escore VISAGE. Pôde-se observar que houve diferença significativa em ambos os grupos: sucesso $(0,001)$ e falha $(0,040)$.

Tabela 3 - Análise estatística da comparação intragrupo, segundo a variável nível de consciência (ECG) no momento da avaliação do escore VISAGE.

\begin{tabular}{lllll}
\hline Variável & Grupos & Inicial & $\begin{array}{l}\text { No momento da } \\
\text { avaliação do Escore }\end{array}$ & p \\
& & & VISAGE & \\
\hline ECG & Sucesso & $8,0[6,0-12,0]$ & $11,0[10,0-11,0]$ & $0,001^{*}$ \\
& Falha & $8,0 \pm 3,5$ & $10,0 \pm 1,0$ & $0,040^{*}$ \\
& & & &
\end{tabular}

ECG: escala de coma de Glasgow. Os resultados estão expressos em média \pm desvio padrão da média ou mediana (intervalos interquartis). Teste Wilcoxon ou teste $\mathrm{T}$ student pareado.

A Tabela 4 apresenta os resultados encontrados na comparação intergrupo das pontuações da ECG inicial versus o momento da avaliação do escore VISAGE. Pôde-se observar que não houve diferença significativa em ambos os grupos estudados.

Tabela 4 - Análise estatística da comparação intergrupo, segundo a variável nível de consciência, avaliada pela Escala de coma de Glasgow (ECG) inicial (no momento da admissão hospitalar) e no momento da avaliação do escore VISAGE.

\begin{tabular}{llll}
\hline \multicolumn{1}{c}{ Variáveis } & \multicolumn{2}{c}{ Grupos } & P \\
\cline { 2 - 3 } & Sucesso $(\mathbf{n}=\mathbf{4 8})$ & Falha $(\mathbf{n}=16)$ & \\
\hline ECG inicial & $8,5 \pm 3,5$ & $8,0 \pm 3,5$ & 0,737 \\
ECG no momento da avaliação & & & \\
do escore de VISAGE & $11,0\lceil 10,0-11,0]$ & $11,0\lceil 10,0-11,0]$ & 0,912 \\
\hline
\end{tabular}

ECG: escala de coma de Glasgow. Os resultados estão apresentados em média \pm desvio padrão da média ou mediana [intervalos interquartis]. Teste T student não pareado ou MannWhitney.

Os resultados referentes à comparação da pontuação total do escore VISAGE e de seus domínios estão apresentados na Tabela 5.

Pôde-se observar que houve diferença significativa entre os grupos em relação ao escore total, sendo que o grupo sucesso obteve valores superiores ( $\mathrm{p}=$ 0,0314 ) ao grupo falha na extubação. Já na comparação das pontuações por domínios entre os dois grupos estudados pôde-se observar que houve diferença significativa apenas no domínio de deglutição $(p=0,007)$.

Tabela 5 - Análise estatística da comparação da pontuação total e dos domínios do Escore VISAGE nos dois grupos estudados.

\begin{tabular}{llll}
\hline Variáveis & \multicolumn{2}{c}{ Grupos } & P \\
\cline { 2 - 3 } & Sucesso (n=48) & Falha (n=16) & 0,031 \\
\hline Escore total do & $2,5[2,0-3,0]$ & $2,0[1,0-3,0]$ & \\
VISAGE & & & 0,082 \\
Idade $>40$ anos) & Sim 35,5 & Sim 12,5 & \\
em \% & Não 64,5 & Não 87,5 & 0,880 \\
Busca visual, em \% & Sim 64,5 & Sim 62,5 & $0,007^{*}$ \\
& Não 35,5 & Não 37,5 & \\
Deglutição, em \% & Sim 87,0 & Sim 56,0 & 0,883 \\
& Não 12,5 & Não 44,0 & \\
ECG $>10$, em \% & Sim 58,5 & Sim 56,0 & \\
& Não 41,5 & Não 44,0 & \\
& & & \\
\hline
\end{tabular}

ECG: Escala de Coma de Glasgow. Os resultados estão em mediana [intervalo interquantis] ou porcentagem. Teste de Mann-Whitney e teste qui-quadrado.

\section{Discussão}

A amostra do presente estudo foi predominantemente composta pelo sexo masculino $(73,44 \%)$, o que epidemiologicamente pode ser explicado devido ao fato de se tratar de um estudo realizado em uma UTI para pacientes vítimas de trauma. Além disso, houve predomínio de pacientes mais jovens entre os participantes que receberam o diagnóstico de TCE. Viégas et al, em $2013^{27}$, demonstram que a maioria das vítimas de TCE é constituída por adultos jovens, do gênero masculino, que se apresentam como mais suscetíveis à violência e à imprudência, assim como aos acidentes de trabalho.

Do mesmo modo que o sexo, a influência da idade no desfecho do desmame da VM, pode variar de acordo com a população a ser estudada ${ }^{8}$. No presente estudo, não foi observado diferença estatisticamente significativa entre a idade dos participantes e o desfecho da retirada da VM. Este resultado se assemelha a outros estudos, apontando que a idade não é um fator preditivo importante no desfecho de desmame da VM em tais pacientes. ${ }^{19,20,22,26}$

Os pacientes neurocríticos apresentam taxas significativamente altas de falha na extubação da VM. De 
acordo com os autores Godet et al, em $2017^{11}$, destacam que essas taxas variam entre $20-40 \%$ nas UTIs neurológicas. Esses resultados estão de acordo com nosso estudo, que mostrou uma taxa de $25,0 \%$ de falha na extubação da VM nos pacientes avaliados.

No presente estudo, não houve diferença proteção das vias aéreas nessa população ${ }^{16}$. Em nosso estudo, significativa entre os pacientes cuja extubação foi realizada o principal motivo de retorno a VM foi a incapacidade de com sucesso e aqueles cuja extubação falhou quanto ao proteção das vias aéreas superiores (56,25\%) entre os pacientes tempo de sedação e tempo de VM, esses achados estão de que apresentaram falha na extubação $(n=16)$.

acordo com alguns autores ${ }^{7,12}$, porém a maioria dos estudos $8,9,13,15$, encontraram resultados opostos, isto é, o maior tempo de ventilação mecânica invasiva (VMI) foi associado aos casos de falha de extubação. Apesar disso, não se pode afirmar que há uma relação de causa e efeito entre o tempo de VMI e a falha de extubação, mesmo porque a maioria dos estudos apresentam outros fatores possivelmente confundidores e não atribuídos a esta população específica.

Igualmente, os resultados do estudo mostraram que a pontuação do APACHE II não teve relação com o sucesso ou insucesso da extubação, pois não houve diferença significativa entre eles, corroborando com o estudo de Amaral et al, em 2016. ${ }^{1}$

Vários autores relatam que o nível de consciência, avaliado isoladamente, não se apresenta como um bom fator preditivo de sucesso no desmame da VM. A maioria dos estudos não mostram um consenso quanto ao nível de consciência mínimo durante o desmame e que não há uma relação bem estabelecida entre o ECG e o sucesso no desmame e retirada da VM nesse perfil de pacientes. ${ }^{16,18,25,26,28}$

Coplin et al. em $2000^{7}$ observaram que pacientes com Escore na Escala de Coma de Glasgow (ECG) $\leqslant 8$ foram extubados com sucesso. Da mesma forma, no estudo de Anderson et al, em $2011^{2}$ os indivíduos que falharam na extubação não apresentaram diferença significativa no ECG quando comparados com aqueles que foram bem-sucedidos. Do mesmo modo, em nosso estudo não foi observada associação significativa entre a pontuação da ECG ao sucesso ou falha na extubação dos pacientes com lesão cerebral, corroborando com os achados na literatura.

Por outro lado, o tempo de internação hospitalar foi maior nos pacientes que tiveram falha na extubação. Os achados sugerem que quanto maior o tempo de internação, mais desfavorável será o desfecho da extubação, reforçando os resultados de outros estudos ${ }^{10,12,21,24}$. Embora outros fatores possam influenciar no tempo de internação, entendemos que a falha de extubação está associada diretamente aos desfechos, visto que tais pacientes, na maioria das vezes, necessitam de retorno à sedação, novo processo de desmame ventilatório, além de estarem mais suscetíveis a complicações inerentes à VMI, como atelectasias e pneumonias. ${ }^{8,9,15,16}$

Referente aos resultados do Escore VISAGE foi observado que o escore pode ser usado como um preditor de sucesso na extubação de pacientes neurológicos em processo de desmame da VM, considerando que a pontuação total do escore foi maior no grupo sucesso na extubação. De acordo com os autores criadores do Escore VISAGE ${ }^{3}$, pontuação igual ou maior a 3 é capaz de predizer o sucesso na extubação em $90 \%$ dos casos de pacientes neurocríticos.

$\mathrm{Na}$ amostra do presente estudo observou-se que na comparação dos domínios avaliados no escore VISAGE, a deglutição foi o único domínio que diferiu entre os dois grupos estudados, ou seja, a capacidade de deglutir foi diretamente relacionada com o desfecho de extubação bem-sucedida.
Sabe-se que pacientes neurocríticos apresentam alta taxa de falha na retirada da VM, e que isso pode estar relacionado mais com a incapacidade de proteção das vias aéreas do que com a capacidade de ventilar espontaneamente 16. Diante disso, há a necessidade em avaliar a capacidade de

A incapacidade de engolir e de depurar as vias aéreas das secreções tem um impacto considerável na capacidade dos pacientes de respirar sem assistência ${ }^{23}$. No estudo de Godet et al. em $2017^{11}$, os autores destacam que a sucedida depende principalmente da avaliação da capacidade de manutenção dos reflexos de proteção das vias aéreas, como a deglutição, e que a presença dela esteve relacionada ao maior sucesso na extubação do paciente neurológico, corroborando com nossos chados.

Contudo, o presente estudo teve limitações por não se tratar de um estudo multicêntrico, o que poderia ter resultado em uma amostra maior.

Concluímos que na amostra de pacientes neurocríticos estudados, valores superiores da pontuação total do escore de VISAGE estiveram associados com o sucesso na extubação, sendo a deglutição um importante fator a ser considerado.

\section{Declaração} interesse.

Os autores declaram ausência de conflito de

\section{Referências}

1. Amaral ERF, Reis HFC. Incidência e impacto clínico da falha de extubação em unidade de terapia intensiva. Bahiana journals. Março de 2016 ;6(2). Disponível em: https://www5.bahiana.edu.br/index.php/fisiotera pia/article/view/808

2. Anderson CD, Bartscher JF, Scripko PD, Biffi A, Chase D, Guanci M, et al. Neurologic examination and extubation outcome in the neurocritical care unit. Neurocrit Care. December 2011;15(3):490-7. Disponível em: https://pubmed.ncbi.nlm.nih.gov/20428967/

3. Asehnoune k, Seguin P, Lasocki S, Roqilly A, Delater A, Gros A, et al. Extubation success prediction in a multicentric cohort of patients with severe brain injury. Anesthesiology. Agoust 2017 ;127(2):338-346. Disponível em: https://pubmed.ncbi.nlm.nih.gov/28640020/

4. Barbas CSV, Isola AM., Farias AMC. Diretrizes Brasileiras de Ventilação Mecânica. AMIB e SBPT. 2013. Disponível em: https://edisciplinas.usp.br/pluginfile.php/23754 4/mod_resource/content/1/Consenso\%20VM\% 202013.pdf. extubação de pacientes com lesão cerebral aguda bem- 
5. Borsellino B, Shultz MJ, Gama de Abreu M, Robba C, Bilotta F, et al. Ventilação mecânica em pacientes com assistência neurocrítica: uma revisão sistemática da literatura. Especialista rev respir med. Setembro $2016 ; 10(10)$ :11231132. Disponível em: https://pubmed.ncbi.nlm.nih.gov/27635737/.

6. Cinotti R, Bouras M, Roquilly A, Asehnoune K. Management and weaning from mechanical ventilation in neurologic patients. Annals of translational medicine. October 2018; 6(19): 381. Disponível em: https://www.ncbi.nlm.nih.gov/pmc/articles/PM C6212362/

7. Coplin WM, Pierson DJ, Cooley KD, Newell DW, Rubenfeld GD. Implications of extubation delay in brain-injured patients meeting standard weaning criteria. Am j respir crit care med. May 2000 ; 161(5):1530-6. Disponível em: https://www.atsjournals.org/doi/full/10.1164/ajr ccm.161.5.9905102

8. Epstein SK. Decision to extubate. Intensive Care Med. 2002 May ;28(5):535-46. Disponível em: https://pubmed.ncbi.nlm.nih.gov/12029399/

9. Esteban A, Frutos-Vivar F, Muriel A, Ferguson ND, Penuelas O, Abraira V, et al. Evolução da mortalidade ao longo do tempo em pacientes recebendo ventilação mecânica. Ats journals. Maio 2013; 188(2): 220-230. Disponível em: https://pubmed.ncbi.nlm.nih.gov/23631814/.

10. Frutos-Vivar F, Esteban A, Apezteguia C, González M, Arabi Y, Restrepo MI, et al. Outcome of reintubated patients after scheduled extubation. J Crit Care. October 2011;26(5):502-509. Disponível em: https://pubmed.ncbi.nlm.nih.gov/21376523/\#: :t ext=Conclusions $\% 3 \mathrm{~A} \% 20 \mathrm{In} \% 20 \mathrm{a} \% 20$ large $\% 20$ cohort,of\%20new\%20complications \%20after\% 20reintubation.

11. Godet T, Chabanne R, Marin J, Kauffmann S, Futier E, Pereira B, et al. Extubation failure in braininjured patients: risk factors and development of a prediction score in a preliminary prospective cohort study. Anesthesiology. January 2017; 126(1):104-114. Disponível em: https://pubmed.ncbi.nlm.nih.gov/27749290/

12. Heubel AD, Mendes RG, Barrile SR, Gimenes C, Martinelli B, Silva LN et al. Falha de extubação em unidade de terapia intensiva pediátrica: estudo de coorte retrospectivo. Fisioter. Pesqui. 2020 Janeiro ; 27(1): 34-40. Disponível em: http://www.scielo.br/scielo.php?script=sci_artte xt\&pid=S1809-29502020000100034\&lng=en.

13. Kavaturo JHHS, Machado FO, Staub LJ, Silva RM. Falha de extubação e suas implicações clínicas em unidade de terapia intensiva. Bol curso med ufsc. Maio 2020 ; 6(1). Disponível em: https://ojs.sites.ufsc.br/index.php/medicina/article /view/3927

14. Ko R, Ramos L. Chalela JA. Parâmetros convencionais de desmame não predizem falha na extubação em pacientes com cuidados neurocríticos. Neurocrit care. Janeiro $2009 ; 10(3): 269-73$. Disponível em: https://pubmed.ncbi.nlm.nih.gov/19184557

15. Kollef MH, Shapiro SD, Silver P, St John RE, Prentice D, Sauer S, Ahrens TS, Shannon W, BakerClinkscale D. A randomized, controlled trial of protocol-directed versus physician- directed weaning from mechanical ventilation. Crit Care Med. April 1997;25(4):567-74. Disponível em: https://pubmed.ncbi.nlm.nih.gov/9142019/.

16. Kutchak FM, Debesaitys AM, Rieder MM, Meneguzzi C, Skueresky AS, Junior LAF, et al. Reflex cough pef as a predictor of successful extubation in neurological patients. Jornal brasileiro de pneumologia. Agoust 2015 ; 41(4):358-364. Disponível em: http://www.scielo.br/scielo.php?Script=sci_arttex t\&amp;pid=s1806- 37132015000400358.

17. Lazaridis C, DeSantis SM, McLawhorn M, Krishna V. Liberation of neurosurgical patients from mechanical ventilation and tracheostomy in neurocritical care. J Crit Care. Agoust;27(4): 417.e1-8. Disponível em: https://pubmed.ncbi.nlm.nih.gov/22033050/

18. Mccredie VA, Ferguson ND, Pinto RL, Adhikari NKJ, Fowler RA, Chapman MG, et al. Airway management strategies for brain-injured patients meeting standard criteria to consider extubation. A prospective cohort study. Ats journals. October 2017;14(1);14:85-93.Disponível em: https://www.atsjournals.org/doi/10.1513/AnnalsA TS.201608-6200C.

19. Navalesi P, Frigerio P, Moretti MP, Sommariva M, Vesconi S, Baiardi $\mathrm{P}$, et al. Rate of reintubation in mechanically ventilated neurosurgical and neurologic patients: evaluation of a systematic approach to weaning and extubation. Crit Care Med. November 2008 ; 36(11):2986-92. Disponível em: https://pubmed.ncbi.nlm.nih.gov/18824909/.

20. Nemer SN, Barbas CS. Predictive parameters for weaning from mechanical ventilation. J Bras Pneumol. September 2011;37(5):669-7. Disponível em:https://pubmed.ncbi.nlm.nih.gov/22042401/\#: : :text=The $\% 20$ most $\% 20$ widely $\% 20$ used $\% 20 \mathrm{w}$ eaning, 1)\%2C\%20the\%20P0.

21. Savi A, Teixeira C, Silva JM, Borges LG, Pereira PA, Pinto KB, Gehm F, Moreira FC, Wickert R, Trevisan CB, Maccari JG, Oliveira RP, Vieira SR; 
Gaúcho Weaning Study Group. Weaning predictors do not predict extubation failure in simple-to-wean patients. J Crit Care. September 2012;27(2): 221.e1-8.Disponível em: https://pubmed.ncbi.nlm.nih.gov/21958979/

22. Seneviratne J, Mandrekar J, Wijdicks EF, Rabinstein AA. Predictors of extubation failure in myasthenic crisis. Arch Neurol. July 2008;65(7):929-33.Disponível em: https://pubmed.ncbi.nlm.nih.gov/18625860/\#: :t ext=Male $\% 20$ sex $\% 2 \mathrm{C} \% 20$ history $\% 20$ of $\% 20$ previous,predicted $\% 20$ the $\% 20$ need $\% 20$ for $\% 20$ r eintubation.

23. Stocchetti, Nino MD; Beretta, Luigi MD; Citerio, Giuseppe MD. Criteria for extubation in neurologic patients, Critical Care Medicine. April 2009 ; 37(4): 1529. Disponível em: https://journals.lww.com/ccmjournal/Citation/20 09/04000/Criteria_for_extubation_in_neurol ogic_patients.71.aspx.

24. Tobin MJ, Jubran A. Variable performance of weaningpredictor tests: role of Bayes' theorem and spectrum and test-referral bias. Intensive Care Med. 2006 December;32(12):2002-12. Disponível em: https://pubmed.ncbi.nlm.nih.gov/17091239/.

25. Vidotto MC, Sogame LC, Calciolari CC, Nascimento OA, Jardim JR. The prediction of extubation success of postoperative neurosurgical patients using frequency-tidal volume ratios. Neurocrit Care. 2008;9(1):83-89. Disponível em: https://pubmed.ncbi.nlm.nih.gov/18250977/

26. Vidotto MC, Sogame LC, Gazzotti MR, Prandini MN, Jardim JR. Analysis of risk factors for extubation failure in subjects submitted to non-emergency elective intracranial surgery.Respir Care. December 2012;57(12):2059 2066. Disponível em: https://pubmed.ncbi.nlm.nih.gov/22613227/

27 Viégas MLC, Pereira ELR, Targino AA, Furtado VG, Rodrigues DB. Traumatismo cranio-encefálico em um hospital de referência no estado do pará, brasil: prevalência das vítimas quanto a gênero, faixa etária, mecanismos de trauma, e óbito. Arq Bras Neurocir. 2013; 32(1):15-8. Disponível em: $\quad$ http://files.bvs.br/upload/s/01035355/2013/v32n1/a3620.pdf.

28. Wendell LC, Raser J, Kasner S, Park S. Predictors of extubation success in patients with middle cerebral artery acute ischemic stroke. Stroke Res Treat. October 2011:248789. Disponível em: https://pubmed.ncbi.nlm.nih.gov/21977336/ 